\title{
Impact of a poor functional capacity on the clinical outcomes in patients with a pacemaker implantation -Results from the Japanese Heart Rhythm Society Registry -
}

Takanori Arimoto ${ }^{1}$, Eiichi Watanabe ${ }^{2}$, Ritsuko Kohno $^{3}$, Kenji Shimeno ${ }^{4}$, Kan Kikuchi ${ }^{5}$, Atsushi Doi ${ }^{6}$, Kanki Inoue ${ }^{7}$, Takashi Nitta ${ }^{8}$, Akihiko Nogami ${ }^{9}$, Haruhiko Abe ${ }^{10}$, and Ken Okumura $^{11}$

${ }^{1}$ Yamagata University School of Medicine

${ }^{2}$ Fujita Health University Bantane Hospital

${ }^{3}$ University of Occupational and Environmental Health

${ }^{4}$ Osaka City General Hospital

${ }^{5}$ Japan Community Healthcare Organization Kyushu Hospital

${ }^{6}$ University Graduate School of Medicine

${ }^{7}$ Sakakibara Heart Institute

${ }^{8}$ Nippon Medical School

${ }^{9}$ Faculty of Medicine University of Tsukuba

${ }^{10}$ University of Occupational and Environmental Health, Japan

${ }^{11}$ Saiseikai Kumamoto Hospital

May 12, 2020

\begin{abstract}
Introduction: To know whether cardiac pacemaker implantations improve the functional capacity (FC) and affect the prognosis. Methods and Results: We prospectively enrolled 621 de-novo pacemaker recipients (age $76 \pm 9$ years, $50.7 \%$ male) between April 2015 and September 2016. The FC was assessed by the metabolic equivalents (METs) during the implantation and periodically thereafter. The patients were a priori classified into a poor FC $(<2$ METs, n=40 [6.4\%]), moderate FC $(24$ METs, n=342 [55.1\%]). Three months after the pacemaker implantation, poor FC or moderate FC patients improved to a good FC by $43 \%$. The distribution of the three FCs remained at those levels by the end of the follow-up $(\mathrm{p}=0.18)$. During a median follow-up of 2.4 years, 71 patients (11\%) had cardiovascular hospitalizations and $35(5.6 \%)$ all-cause death. A multivariate Cox analysis revealed that a poor FC at baseline was an independent predictor of both a cardiovascular hospitalization (hazard ratio [HR] 2.494, 95\% confidence interval [CI $1.227-5.070, \mathrm{p}=0.012$ ) and all-cause death (HR 3.338, 95\% CI 1.254-8.886, p=0.016). One year after the pacemaker implantation, the 19 patients whose poor FC improved to a good FC did not die, however, the 8 who remained with a poor FC had a high mortality rate of $37.5 \%(\mathrm{p}<0.01)$. Conclusion: Approximately half of the poor or moderate FC patients improved to a good FC 3 months after the pacemaker implantation. The baseline FC predicted the prognosis, and patients with an improved FC after the pacemaker implantation had a better prognosis.
\end{abstract}

\section{Introduction}

Patients with bradyarrhythmias undergo [?]1 million de novo pacemaker implantations annually worldwide. ${ }^{1}$ Since the first pacemaker implantations almost 60 years ago, permanent cardiac pacemaker 
therapy has evolved remarkably, becoming a minimally invasive treatment, improving the quality of life and reducing the mortality. ${ }^{1-3}$ Device implantations are now indicated not only for young and middle-aged individuals who need to maintain physical activity, but also for elderly patients and those with a reduced physical function. There is wide recognition that the $\mathrm{FC}$ in patients with various cardiovascular diseases is an important risk factor for worsening heart failure and an increased risk of mortality, ${ }^{4,5}$ however, there are limited data on whether pacemaker implantations improve the FC, and whether changes in the FC affect the outcomes.

In Japan, there is a system that exempts patients with serious diseases from medical expenses as handicapped disabled patients. In the case of patients with an initial pacemaker implant, the handicapped disability levels can be divided into three levels depending on the indication of the pacemaker implantation defined by the Japanese Circulation Society ${ }^{6}$ and their FC is determined by the metabolic equivalents (METs). Currently, three years after implanting a pacemaker, the disability level is recertified based on the FC at that time. However, it is not known how the FC changes over time after the initial pacemaker implantation, and therefore, the optimal time for the recertification should be determined by prospective studies. Therefore, the aim of this study was to examine the temporal trends in the FC after a pacemaker implantation and the relationship between the FC and prognosis in patients receiving a de novo pacemaker implantation.

\section{Methods}

\section{Study Population}

This registry was a prospective, multicenter registry enrolling patient receiving de novo pacemaker implantations at 28 centers in Japan from April 2015 to September 2016. Consenting patients were enrolled if they were at least 20 years old and had a pacemaker indication according to the Japanese guidelines. ${ }^{6}$ Patients were excluded if they refused participation in this study. Informed consent was obtained from all patients prior to participation, and the study protocol was approved by each institutional Human Investigations Committee. The investigation was performed in accordance with the ethical standards as laid down in the 1964 Declaration of Helsinki and its later amendments.

\section{Data Collection and Definitions}

The patient characteristics and baseline and follow-up data were obtained through a review of their hospital charts. The anonymized patient data were collected in spreadsheet format by the physicians or clinical research coordinators at each institution. We examined the demographics, etiology of the pacemaker implantation, class of the JCS guideline indication, ${ }^{6}$ and history of heart failure. The history of heart failure was determined as acute heart failure or worsening of chronic heart failure requiring hospitalization. The $\mathrm{FC}$ was estimated from the interview of the activities of daily living using a questionnaire, 7,8 and it was a prioriclassified into a poor FC $(<2$ METs $)$, moderate FC $(2<$ METs $<4)$, and good FC $(>4$ METs $)$ (Supplementary file ).

\section{Follow-up and Endpoints}

After the implantation, the patients were followed up at each hospital once every few months for 6 months, and thereafter once every 6 months. The FC was recorded at 3 months, 6 months, and 1 year after the pacemaker implantation. The endpoints were cardiovascular hospitalization and all-cause mortality.

\section{Statistical Analysis}

Continuous variables are expressed as the mean $\pm \mathrm{SD}$ or median with the interquartile range. Continuous and categorical variables were compared with a Student's t-test and $\chi^{2}$ test, respectively. Univariate and 
multivariate analyses with a Cox proportional hazard regression model was used to identify the significant predictors of the outcomes. The multivariate Cox proportional hazard analysis adjusted for the age, sex, and significant variables in the univariate analyses, and a history of heart failure and atrial fibrillation. The event-free curves were computed using the Kaplan-Meier method and compared with a log-rank test. A P < 0.05 was considered statistically significant. We used JMP version 11.0 software (SAS Institute Inc., Cary, NC, USA).

\section{Results}

\section{Patient characteristics}

All patients underwent a successful pacemaker implantation, and there were no complications or deaths 30 days post-procedure. The baseline characteristics of the patients are shown in Table 1 . The mean age was $76 \pm 9$ years (range 29 to 98 years), and there were 315 males (50.7\%). The etiology of the device implantation was identified as atrioventricular block in $307(49.4 \%)$ patients, sick sinus syndrome in 276 $(44.4 \%)$, atrial fibrillation in $32(5.2 \%)$, and others in the remaining $6(1.0 \%)$. At the time of the device implantation, 583 (93.9\%) patients were diagnosed with a grade I disability due to a JCS guideline class I indication. A history of heart failure was noted in 238 (38.3\%) patients.

\section{Changes in the functional capacity}

The distribution of the FC at baseline, 3 months, 6 months, and 1 year after the pacemaker implantation is shown in Figure 1. Three months after the pacemaker implantation, 16 patients with a poor FC and 105 with a moderate FC at baseline improved to a good FC (Figure 1A ). However, 3 patients with a moderate FC and 4 with a good FC at baseline deteriorated to a poor FC at 3 months after the implantation. Three months after the pacemaker implantation, the distribution of the three FCs remained at those levels by the end of the follow-up $(\mathrm{p}=0.18)$.

\section{Predictors of cardiovascular hospitalizations and all-cause mortality}

During a median of 2.4 years of follow-up (interquartile range 0.2 to 3 years), 71 (11\%) patients had a cardiovascular hospitalization (heart failure $[n=45]$, ischemic heart disease $[n=18]$, and strokes $[n=8]$ ). A total of 35 patients $(5.6 \%)$ died due to a malignancy $(n=8)$, sepsis $(n=7)$, heart failure $(n=6)$, respiratory failure $(n=3)$, sudden death $(n=2)$, stroke $(n=1)$, or other causes $(n=8)$. The univariate Cox analysis revealed that a history of heart failure and a poor FC were significantly associated with a hospitalization (Table 2 ). The multivariate Cox analysis adjusted for the age, sex, history of heart failure, and a poor FC revealed that a history of heart failure (HR 2.097, 95\% CI 1.275-3.448, $\mathrm{p}=0.004$ ) and a poor FC (HR 2.494, 95\% CI 1.227-5.070, $\mathrm{p}=0.012$ ) remained as independent predictors. For the all-cause death, the age (HR 1.096, 95\% CI 1.043-1.151, p<0.001) and a poor FC (HR 3.338, 95\% CI 1.254-8.886, p=0.016) were independent predictors after being adjusted for the age, sex, and a poor FC (Table 3 ). The Kaplan-Meier analysis demonstrated that the rate of a hospitalization (Figure 2A ) and the total mortality were significantly higher as the FC decreased (Figure 2B ).

\section{Subgroup analysis of poor functional capacity patients 1 year after the pacemaker implantation}

We further examined the outcome of the 40 patients with a poor FC at the time of the pacemaker implantation (Figure. 3A ). Three patients died and 3 were lost to follow-up at 1 year after the device implantation. Of the remaining 34 patients, $8(20 \%)$ had no improvement in the FC, but 7 and 19 patients improved to a moderate FC and a good FC, respectively. Those 34 patients were further followed for a median of 2 
years (interquartile range 0.3 to 2 years). The patients that remained with a poor FC one year after the pacemaker implantation had a significantly higher mortality rate $(37.5 \%)$ than those that improved to a good FC (Figure 3B ).

\section{Discussion}

We examined the temporal trends in the FC and the relationship between the FC and prognosis in patients receiving an initial pacemaker implantation. The major findings of this study were that (1) $43 \%$ of the patients with a poor or moderate FC improved to a good FC 3 months after the pacemaker implantation, (2) a poor FC was an independent predictor of both a hospitalization and the total mortality, and (3) the lack of an improvement in the FC at 1 year after the pacemaker implantation was associated with an increased risk of mortality.

It is well established that the exercise capacity, daily physical activity, and daily walking performance are significantly associated with the clinical outcome. ${ }^{9-11}$ Recent studies using pacemaker built-in accelerometers found a significant correlation between the physical activity and mortality. Tyagi et al. assessed the physical activity measured by a pacemaker accelerometer in de novo pacemaker implantation patients who had a preserved left ventricular function. ${ }^{12}$ They classified the patients into 4 groups based on the average active time and followed them for an average of 4.1 years. The rate of the all-cause mortality significantly increased as the active minutes decreased. Patients with an average of $<1 \mathrm{~h}$ /day of active time had a nearly 7.5fold increased risk of death compared to those who were active $>3 \mathrm{~h} /$ day. In another study, Conraads et al. showed a significant relationship between the physical activity and the mortality in patients with implantable cardioverter defibrillators (ICDs) and reduced the left ventricular ejection fraction. The patients in the lowest tertile for daily activity $(<146 \mathrm{~min} /$ day) had a 5 times higher risk of mortality compared to those in the highest tertile for daily activity (>235 min/day). ${ }^{13}$ These data suggested that the device-measured physical activity may have served as a marker for the unmeasured factors contributing to the mortality risk. In addition, a novel chronotropic incompetence measure (Heart Rate Score) also predicts a worse outcome in patients that undergo device implantations. ${ }^{14,}{ }^{15}$ Furthermore, Richards et al. suggested that a blended sensor with minute ventilation and an accelerometer improves the Heart Rate Score in patients with pacemakers. ${ }^{16}$ While these device built-in objective indicators may be useful to quantify an individual's activity, this device software has difficulty in interpreting because the measurement method and calculation method differ depending on the manufacturer. Additionally, device interrogation or remote monitoring are required to use these indicators. On the other hand, a subjective FC by a questionnaire, not a device-measured physical activity, is easily obtainable and is able to predict the outcome.

In this study we showed that $43 \%$ of the patients with a poor or moderate $\mathrm{FC}$ at the time of the pacemaker implantation had an improved FC 3 months after the implantation and an improved FC was associated with a better outcome. Previous studies showed that the device-measured physical activity increased over a 30-day period after the implantation in patients that received an ICD or cardiac resynchronization therapy defibrillator. ${ }^{13}$ That observation was consistent with our study, in that a most of the patients had an improved FC probably because the bradycardia-associated symptoms were alleviated by the pacemaker. Fleischmann et al. demonstrated that the pacemaker implantation itself was associated with a significant improvement in the health-related quality of life (QOL) scores. This improvement extended to almost all domains such as the physical function, physical role, social function, mental health, and vitality. ${ }^{17}$ Importantly, a QOL improvement was similarly observed irrespective of the sex, presence of heart failure, or comorbidity level. We suspected that the improved QOL with the pacemaker implantation may also have influenced the subsequent prognosis in the present study.

To preserve or improve the physical activity after the permanent pacemaker implantation, physiological pacing is expected to be a promising strategy. Minimizing any inadvertent ventricular pacing is important for maintaining the exercise capacity and preventing subsequent cardiac events. A more physiological pacing such as His-bundle pacing ${ }^{18}$ is a promising option. Among patients with left ventricular (LV) systolic dysfunction 
and a wide QRS complex or with ventricular pacing dependency, cardiac resynchronization therapy may be a better device to improve the exercise tolerance. An improvement in the hemodynamic profile and sympathoinhibitory effect lead to a reversal of skeletal myopathy and an enhanced exercise performance. Cardiac resynchronization increases the LV contractility and reduces functional mitral regurgitation, resulting in an increased cardiac output and $\mathrm{dp} / \mathrm{dt}$ index. These improvements in the cardiac hemodynamics result in a decrease in the muscle sympathetic nerve activity, reversal of muscle inflammation, and improve the long-term skeletal myopathy. ${ }^{19}$

Our study showed that physical inactivity was associated with a poor survival independent of other risk factors. Cardiologists should pay attention not only to the device condition but also to encourage increased physical activity and to follow the patient compliance with physical activity recommendations. Considering the association between significantly reduced physical activity and a poor prognosis, individuals with an FC of $<2$ METs were stratified into serious conditions and required careful observation. In the majority of patients, the FC improved at 3 months after the pacemaker implantation and was maintained for at least 1 year. Patients with an improved FC at 1 year after the pacemaker implantation had a relatively good prognosis. Particularly, no patients died in the group with an improved FC (METs $>4$ ). Thus, a reevaluation of the physical disability between 3 months and one year may be suitable in patients who have undergone a pacemaker implantation.

\section{Study Limitations}

Our study included heterogenous patients receiving pacemakers for atrioventricular block, sick sinus syndrome, and atrial fibrillation with a slow ventricular response. We did not investigate the detailed pacemaker pacing mode, pacing rate, heart rate distribution (i.e., Heart Rate Score), or pacing site, therefore, the relationship between the pacemaker settings and physical activity could not be examined. However, irrespective of the device status, a poor FC was proven to be useful as a prognostic indicator. The conventional prognostic clinical tests such as the B-type natriuretic peptide, renal function, echocardiographic findings, and cardiopulmonary exercise testing were not analyzed. This study did not include the currently available leadless pacemakers.

\section{Conclusions}

The pacemaker implantation improved the FC in $43 \%$ of the patients with a poor or moderate $\mathrm{FC}$ at baseline and remained at that level to the end of 1 year. The poor FC $(<2$ METs $)$ at baseline was significantly associated with a worse outcome. The patients whose FC improved at 1 year after the pacemaker implantation had a relatively good prognosis.

\section{Acknowledgement}

The authors would like to thank Ms. Yoko Sato of the Japanese Heart Rhythm Society for her assistance in the data management.

\section{Disclosures}

Financial support: This study was partially supported by a research grant from the Japanese Ministry of Health, Labour and Welfare.

Conflict of interest . EW received a lecture fee from Biotronik Japan, Daiichi-Sankyo, and Pfizer. RK has an affiliation with the endowed departments of Boston Scientific and Abbott. NA received a lecture fee from Daiichi Sankyo and has an affiliation with the endowed department of Boston Scientific and Abbott. KO received a lecture fee from Johnson and Johnson, Medtronic, Daiichi-Sankyo, and Boehringer-Ingelheim. None of the other authors have conflicts of interest.

Data available on request 
The data underlying this article will be shared on reasonable request to the corresponding author.

\section{Author contributions}

Takanori Arimoto Data analysis, Drafting article

Eiichi Watanabe Data collection, Data analysis, Critical revision of article

Ritsuko Kohno Data collection, Approval of article

Kenji Shimeno Data collection, Approval of article

Kan Kikuchi Data collection, Approval of article

Atsushi Doi Data collection, Approval of article

Kanki Inoue Data collection, Approval of article

Takashi Nitta Data collection, Approval of article

Akihiko Nogami Data collection, Approval of article

Haruhiko Abe Data collection, Concept/design, Critical revision of article

Ken Okumura Data collection, Concept/design

\section{References}

1. Epstein AE, DiMarco JP, Ellenbogen KA, Estes NA 3rd, Freedman RA, Gettes LS, Gillinov AM, Gregoratos G, Hammill SC, Hayes DL, Hlatky MA, Newby LK, Page RL, Schoenfeld MH, Silka MJ, Stevenson LW, Sweeney MO, Smith SC Jr, Jacobs AK, Adams CD, Anderson JL, Buller CE, Creager MA, Ettinger SM, Faxon DP, Halperin JL, Hiratzka LF, Hunt SA, Krumholz HM, Kushner FG, Lytle BW, Nishimura RA, Ornato JP, Page RL, Riegel B, Tarkington LG, Yancy CW. American College of Cardiology/American Heart Association Task Force on Practice G, American Association for Thoracic S and Society of Thoracic S. ACC/AHA/HRS 2008 Guidelines for Device-Based Therapy of Cardiac Rhythm Abnormalities: a report of the American College of Cardiology/American Heart Association Task Force on Practice Guidelines (Writing Committee to Revise the ACC/AHA/NASPE 2002 Guideline Update for Implantation of Cardiac Pacemakers and Antiarrhythmia Devices): developed in collaboration with the American Association for Thoracic Surgery and Society of Thoracic Surgeons.Circulation . 2008;117:e350-408. doi: 10.1161/CIRCUALTIONAHA.108.189742.

2. Toff WD, Camm AJ, Skehan JD, United Kingdom P and Cardiovascular Events Trial I. Single-chamber versus dual-chamber pacing for high-grade atrioventricular block. $N$ Engl $J$ Med . 2005;353:145-55. doi: 10.1056/NEJMoa042283.

3. Andersen HR, Nielsen JC, Thomsen PE, Thuesen L, Mortensen PT, Vesterlund T, Pedersen AK. Longterm follow-up of patients from a randomised trial of atrial versus ventricular pacing for sick-sinus syndrome. Lancet . 1997;350:1210-6. doi: 10.1016/S0140-6736(97)03425-9.

4. Tsutsui H, Isobe M, Ito H, Okumura K, Ono M, Kitakaze M, et al. Japanese Circulation S and the Japanese Heart Failure Society Joint Working G. JCS 2017/JHFS 2017 Guideline on Diagnosis and Treatment of Acute and Chronic Heart Failure- Digest Version. Circ J . 2019;83:2084-2184. doi: 10.1253/circj.CJ-19-0342.

5. Kamiya K, Yamamoto T, Tsuchihashi-Makaya M, Ikegame T, Takahashi T, Sato Y, Kotooka N, Saito Y, Tsutsui H, Miyata H, Isobe M. Nationwide Survey of Multidisciplinary Care and Cardiac Rehabilitation for Patients With Heart Failure in Japan- An Analysis of the AMED-CHF Study. Circ J . 2019;83:1546-1552. doi: 10.1253/circj.CJ-19-0241.

6. 2018 JCS/JHRS Guideline on Non-Pharmacotherapy of Cardiac Arrhythmias. 2019;2020. 
7. Hlatky MA, Boineau RE, Higginbotham MB, Lee KL, Mark DB, Califf RM, F R Cobb FR, Pryor DB. A brief self-administered questionnaire to determine functional capacity (the Duke Activity Status Index). Am J Cardiol . 1989;64:651-4. doi: 10.1016/0002-9149(89)90496-7.

8. Ainsworth BE, Haskell WL, Whitt MC, Irwin ML, Swartz AM, Strath SJ,O'Brien WL, Bassett Jr DR, Schmitz KH, Emplaincourt PO, Jacobs Jr DR, Leonet AS. Compendium of physical activities: an update of activity codes and MET intensities. Med Sci Sports Exerc . 2000;32:S498-504. doi: 10.1097/00005768200009001-00009.

9. Blair SN, Kohl HW, 3rd, Barlow CE, Paffenbarger RS, Jr., Gibbons LW and Macera CA. Changes in physical fitness and all-cause mortality. A prospective study of healthy and unhealthy men. JAMA . 1995;273:1093-8.

10. Myers J, Prakash M, Froelicher V, Do D, Partington S and Atwood JE. Exercise capacity and mortality among men referred for exercise testing. $N$ Engl J Med . 2002;346:793-801. doi: 10.1056/NEJMoa011858.

11. Jehn M, Schmidt-Trucksass A, Schuster T, Weis M, Hanssen H, Halle M, Koehler F. Daily walking performance as an independent predictor of advanced heart failure: Prediction of exercise capacity in chronic heart failure. Am Heart J . 2009;157:292-8. doi: 10.1016/j.ahj.2008.10.006.

12. Tyagi S, Curley M, Berger M, Fox J, Strath SJ, Rubenstein J, Roth J, Widlansky ME. Pacemaker quantified physical activity predicts all-cause mortality. J Am Coll Cardiol . 2015;66:754-5. doi: 10.1016/j.jacc.2015.06.005.

13. Conraads VM, Spruit MA, Braunschweig F, Cowie MR, Tavazzi L, Borggrefe M, Hill MR, Jacobs S, Gerritse B, van Veldhuisenet DJ. Physical activity measured with implanted devices predicts patient outcome in chronic heart failure. Circ Heart Fail . 2014;7:279-87. doi: 10.1161/CIRCHEARTFAILURE.113.000883.

14. Olshansky B, Richards M, Sharma A, Wold N, Jones P, Perschbacher D, Wilkoff BL. Survival after rate-responsive programming in patients with cardiac resynchronization therapy-defibrillator implants is associated with a novel parameter: The Heart Rate Score. Circ Arrhythm Electrophysiol . 2016;9:e003806. doi: 10.1161/CIRCEP.115.003806.

15. Wilkoff BL, Richards M, Sharma A, Wold N, Jones P, Perschbacher D, Olshansky B. A device histogrambased simple predictor of mortality risk in ICD and CRT-D patients: The Heart Rate Score. Pacing Clin Electrophysiol . 2017;40:333-343. doi: 10.1111/pace.13036.

16. Richards M, Olshansky B, Sharma AD, Wold N, Jones P, Perschbacher D, Wilkoff BL. Addition of minute ventilation to rate-response pacing improves heart rate score more than accelerometer alone. Heart Rhythm . 2018;15:1730-1735. doi: 10.1016/j.hrthm.2018.06.021.

17. Fleischmann KE, Orav EJ, Lamas GA, Mangione CM, Schron E, Lee KL, Goldman L. Pacemaker implantation and quality of life in the Mode Selection Trial (MOST). Heart Rhythm

2006;3:653-9. doi: 10.1016/j.hrthm.2006.02.1031.

18. Sharma PS, Dandamudi G, Naperkowski A, Oren JW, Storm RH, Ellenbogen KA, Vijayaraman P. Permanent His-bundle pacing is feasible, safe, and superior to right ventricular pacing in routine clinical practice.Heart Rhythm . 2015;12:305-12. doi: 10.1016/j.hrthm.2014.10.021.

19. Iliou MC, Blanchard JC, Lamar-Tanguy A, Cristofini P and Ledru F. Cardiac rehabilitation in patients with pacemakers and implantable cardioverter defibrillators. Monaldi Arch Chest Dis . 2016;86:756. doi: 10.4081/monaldi.2016.756.

Figure legends

Figure 1. The time course of the functional capacity. (A) Changes in the functional capacity between baseline and 3 months after the pacemaker implantation. Three months after the pacemaker implantation, $43 \%$ of those with a poor and moderate FC at baseline improved to a good FC $(16+105 / 40+239)$. (B) The 
distribution of the FCs at baseline, 3 months, 6 -month, and 1 year after the pacemaker implantation. The patients were $a$ priori classified into a poor FC $(<2 \mathrm{METs})$, moderate $\mathrm{FC}(2<\mathrm{METs}<4)$, and good FC $(>$ 4 METs). $\mathrm{FC}=$ functional capacity, METs=metabolic equivalents.

Figure 2 . Kaplan-Meier analysis of the time to the subsequent outcome.

(A ) The cardiovascular hospitalization rate was higher in the patients with a poor FC than in those with a moderate or good FC.

(B ) The total mortality rate was higher in the patients with a poor FC than in those with a moderate FC or good FC. FC=functional capacity.

Figure 3 . Subgroup analysis among the patients with a poor functional capacity.

(A ) Changes in the functional capacity during a 1-year follow-up. Seven (1.75\%) patients improved to a moderate FC, and 19 (47.5\%) had a marked improvement. FC=functional capacity, F/U=follow up.

(B) The total mortality rate. A log-rank test was not applied because no patients with a good FC died.

\section{Hosted file}

Figure for JCE.pptx available at https://authorea.com/users/321197/articles/450488-impactof-a-poor-functional-capacity-on-the-clinical-outcomes-in-patients-with-a-pacemakerimplantation-results-from-the-japanese-heart-rhythm-society-registry 